\title{
A AUTO-PERCEPÇÃO DA SAÚDE AUDITIVA E VESTIBULAR DE TRABALHADORES EXPOSTOS A ORGANOFOSFORADOS
}

\author{
Auto-perception of auditory and vestibular health \\ in workers exposed to organophosphate
}

\author{
Ana Cristina Hiromi Hoshino ${ }^{(1)}$, Heloisa Pacheco-Ferreira ${ }^{(2)}$, Carlos Kazuo Taguchi (3), \\ Shiro Tomita ${ }^{(4)}$, Maria de Fátima Miranda ${ }^{(5)}$
}

\begin{abstract}
RESUMO
Objetivo: caracterizar os sintomas auditivos e vestibulares de trabalhadores rurais expostos aos agrotóxicos organofosforados. Métodos: foi realizado um estudo epidemiológico descritivo com uma amostra de 50 trabalhadores rurais. A faixa etária variou de 21 a 59 anos, média de 38,3 anos, sendo $20(40 \%)$ trabalhadores de sexo masculino e $30(60 \%)$ de sexo feminino. Foi utilizado um questionário com perguntas relacionadas à saúde auditiva e dados sobre tempo de exposição ao agrotóxico. Resultados: os resultados mostraram que 38 trabalhadores $(76 \%)$ referiram ter apresentado pelo menos um episódio de tontura em sua vida e destes, 29 (58\%) trabalhadores ainda sentem tontura; 27 (54\%) sentem zumbidos; 23 (46\%) sentem a orelha abafada; 37 (74\%) acham que possuem boa acuidade auditiva, porém 35 (70\%) acham que, sentem dificuldades na compreensão de palavras, sugerindo que os agrotóxicos podem induzir alterações do sistema auditivo e vestibular por meio de uma intoxicação lenta e silenciosa. Conclusão: a tontura e a perda auditiva aparecem como sintomas subjetivos e constantes da exposição ocupacional podendo ser um sinal precoce da intoxicação, prejudicando a qualidade de vida destes trabalhadores.
\end{abstract}

DESCRITORES: Exposição Ambiental; Riscos Ocupacionais; Inseticidas Organofosforados; Tontura

\section{INTRODUÇÃO}

O aumento do uso de substâncias químicas no meio ambiente tem acarretado graves conse-

(1) Fonoaudióloga; Clínica Otorrino Barra, RJ; Mestre em Saúde Pública pelo Instituto de Estudos em Saúde Coletiva da Universidade Federal do Rio de Janeiro.

(2) Médica; Professora Adjunta do Instituto de Estudos em Saúde Coletiva da Universidade Federal do Rio de Janeiro, UFRJ, Rio de Janeiro, RJ; Doutora em Desenvolvimento Sustentável do Trópico Úmido pela Universidade Federal do Pará.

(3) Fonoaudiólogo; Professor Adjunto da Faculdade de Ciências Médicas da Santa Casa de São Paulo, FCMSCSP, São Paulo, SP; Doutor em Distúrbios da Comunicação Humana pela Universidade Federal de São Paulo.

(4) Médico; Professor Titular da Faculdade de Medicina da Universidade Federal do Rio de Janeiro, UFRJ, Rio de Janeiro, RJ; Doutor em Medicina (Otorrinolaringologia) pela Universidade Federal de São Paulo.

(5) Fonoaudióloga; Professora Auxiliar do Curso de Fonoaudiologia da Universidade Federal do Rio de Janeiro, UFRJ, Rio de Janeiro, RJ; Especialista em Patologia da Linguagem pela Universidade Católica de Pernambuco.

Conflito de interesses: inexistente quências tanto no meio ambiente quanto na população produtiva exposta. Se por um lado, o desenvolvimento tecnológico avança a passos largos, por outro, as pesquisas acerca dos efeitos de tais tecnologias sobre a saúde humana e do meio ambiente não se desenvolvem com tanta rapidez. Considerando-se que as pesquisas se iniciam a partir de um fato gerador, entende-se que o desconhecimento inicial do impacto dessas substâncias químicas no mundo pode ser muito mais nocivo e danoso do que o esperado. Uma vez que a legislação também é lenta, os efeitos maléficos já instalados causam problemas de saúde à sociedade e degradação do meio ambiente, inclusive para as futuras gerações. Uma vez que os dados confiáveis sobre o possível impacto de uma substância danosa ao meio ambiente e à saúde humana estejam disponíveis, não obstante ainda haver incerteza científica, deve-se tomar uma decisão baseada no princípio da precaução (Comissão das Comunidades Europeias, 2001) ${ }^{1}$.

Anualmente, três milhões de pessoas são contaminadas por agrotóxicos em todo o mundo, sendo 
$70 \%$ desses casos nos países em desenvolvimento ${ }^{2}$, onde o difícil acesso às informações e à educação por parte dos usuários desses produtos, bem como o baixo controle sobre sua produção, distribuição e utilização são alguns dos principais determinantes na constituição dessa situação como um dos importantes desafios de saúde pública.

As exposições podem ser divididas em ocupacionais e ou ambientais. Dentro do grupo dos expostos ocupacionalmente encontram-se os agricultures, trabalhadores de indústrias químicas, aplicadores de controle de vetores (como o Aedes aegypti), entre outros. Já com relação às exposições ambientais, estão as residências, produtos de limpeza e dedetização, alimentação (principalmente de neonatos e crianças) e até armas químicas ${ }^{3}$.

Essa exposição produz alterações clínicas que não são detectadas no exame neurológico e nem pelos marcadores biológicos e que, silenciosamente, modificam a vida do trabalhador. Os sintomas subclínicos, considerados como sinal precoce da intoxicação ${ }^{4,5}$, podem ser avaliados por meio de exames neurofisiológicos e neuropsicológicos ${ }^{6}$, apresentando alterações tanto do sistema nervoso periférico como central ${ }^{7,8}$; sendo a tonteira um sintoma subclínico como consequência da exposição aguda ou longo prazo ao agrotóxico amplamente divulgada na literatura ${ }^{\text {9-17. }}$.

Uma substância pode ter seu efeito potencializado se estiver combinada com outra substância tóxica ou em condições favoráveis como variação de temperatura ou ambiente (água, ar, etc), elevando sua concentração e aumentando seu grau de toxicidade. As manifestações clínicas mais comuns devido à exposição aos agrotóxicos são dor de cabeça, distúrbios gastrintestinais e tonteira ${ }^{18}$.

Um estudo de prevalência com 98 trabalhadores expostos aos agrotóxicos organofosforado ${ }^{19}$ observou que $35,7 \%$ sentem tonteiras, $46 \%$ dificuldades em compreender o que falam e $24 \%$ zumbidos. $63,8 \%$ dos expostos apenas aos inseticidas apresentaram perda auditiva; para o grupo com exposição concomitante aos inseticidas e ruído, a perda auditiva foi de $66,7 \%$. Os autores concluíram que há evidência de que a exposição aos agrotóxicos induz dano auditivo periférico e que o ruído é um fator que interage com os agrotóxicos, potencializando seus efeitos ototóxicos.

O reconhecimento da intoxicação por agentes químicos é a chave para um diagnóstico rápido e preciso ${ }^{20}$, portanto o objetivo desse estudo foi caracterizar os sintomas auditivos dos trabalhadores expostos ao agrotóxico organofosforado.

\section{MÉTODOS}

O presente estudo, do tipo transversal com caráter epidemiológico descritivo foi desenvolvido por meio da análise de um questionário (Figura 1) respondido voluntariamente por 50 trabalhadores rurais que faziam parte de uma associação de trabalhadores do Município de Teresópolis, estado do Rio de Janeiro, localizada especificamente na região de Córrego das Pedras e Baú.

A faixa etária verificada variou de 21 a 59 anos, sendo $20(40 \%)$ sujeitos do sexo masculino e 30 $(60 \%)$ do sexo feminino.

Quanto aos critérios de inclusão, foram considerados os sujeitos trabalhadores expostos ao organofosforado e que apresentaram algum episódio de tontura ou apresenta tontura. Foram excluídos aqueles que apresentassem fatores hereditários e/ou congênitos para perda auditiva, história pregressa de otites de repetição, perda de audição decorrente de infecções pós-natal como meningite e parotidite endêmica, e alterações anatômicas de orelha externa e/ou média. As variáveis idade e sexo não foram determinadas.

Os dados foram obtidos por meio da aplicação de um questionário, no qual foram incluídas perguntas pertinentes ao critério de inclusão/exclusão da pesquisa, dados sobre saúde do sistema auditivo e vestibular, e tempo de exposição ao agrotóxico. Serão descritos também, os agrotóxicos mais utilizados pelos trabalhadores, mostrando o grau de periculosidade de cada um.

O estudo considerou os aspectos éticos recomendados pela Resolução 196/96 sobre Pesquisa envolvendo seres humanos, incluindo entre outros, a obtenção do Consentimento Livre e Esclarecido dos indivíduos. Assegurou que a participação não acarretaria nada que pudesse levar a danos físicos, psíquicos, morais, intelectuais, sociais, culturais ou espirituais dessas pessoas. $O$ estudo foi avaliado e aprovado (Processo n. 38/2005) pelo Comitê de Ética do Instituto de Estudo de Saúde Coletiva (IESC/UFRJ).

\section{RESULTADOS}

A partir das respostas dos questionários foi possível organizar a Tabela 1, contendo as referências de uso dos agrotóxicos mais usados pelos sujeitos da pesquisa e sua classificação toxicológica segundo o Ministério da Agricultura/Ministério da Saúde, baseada no Decreto 98.816/90 de 11 de janeiro de 1990, que regulamenta a Lei 7.802/89 de acordo com sua potencialidade tóxica. 


\section{QUESTIONÁRIO}

Número

Nome

Idade End

Tel de contato

1- Você já sentiu tonteira e/ou desequilíbrio ?

( ) $\operatorname{sim}$ ( ) não

2- Você sente atualmente tonteira e/ou desequilíbrio ?

( ) sim ( ) não

3- Você sente zumbidos, barulhos no ouvido ?

( ) $\operatorname{sim}$ ( ) não

4- Você sente os ouvidos tapados ?

( ) $\operatorname{sim}$ ( ) não

5- Você acha que escuta bem ?

( ) $\operatorname{sim}$ ( ) não

6- Você acha que escuta mas às vezes não compreende o que falam ?

( ) $\operatorname{sim}$ ( ) não

7- Você já teve algum problema no ouvido?

( ) $\operatorname{sim}($ ) não

( ) infecções ( ) dores ( ) cirurgias ( ) perfurações( ) outros

8- Qual é a sua atividade profissional ?

9- Você trabalha a quantos anos nesta profissão ?

10- Qual o agrotóxico (veneno) que você usa em cada cultura ?

Figura 1-Questionário respondido pelos 50 trabalhadores expostos ao organofosforado. Teresópolis/ RJ, 2006

O agrotóxico mais utilizado pelos agricultores foi o Manzate $(54,2 \%)$ cuja classificação toxicológica é III, seguido do Folidol (33,3\%), Tamaron $(27,1 \%)$ e Gramoxone (25\%), cujas classificações toxicológicas foram do tipo I, considerado altamente tóxico.

A Tabela 2 apresenta dados sobre a população pesquisada quanto sexo, a distribuição da faixa etária e tempo de trabalho.
De acordo com a Tabela 2, observou-se que 20 trabalhadores foram de sexo masculino $(40 \%)$ e 30 de sexo feminino $(60 \%)$. A idade variou entre 21 a 59 anos, sendo que a maior prevalência de trabalhadores esteve na faixa etária de 21 a 35 anos $(44,0 \%)$. 
Tabela 1 - Descrição dos agrotóxicos mais utilizados pelos 50 trabalhadores expostos ao organofosforado, que responderam o Questionário I. Teresópolis/RJ, 2006

\begin{tabular}{ccccc}
\hline $\begin{array}{c}\text { NOME } \\
\text { COMERCIAL }\end{array}$ & PRINCíPIO ATIVO & $\begin{array}{c}\text { CLASSE } \\
\text { TOXICOLÓGICA }\end{array}$ & N & \% \\
\hline Tamaron & Metamidofós & I & 13 & 27,1 \\
Gramoxone & Paraquat & I & 12 & 25,0 \\
Folidol & P-03 Paration & I & 15 & 33,3 \\
Decis & Deltametrina & II & 6 & 12,1 \\
Manzate & M-03 maneb & III & 26 & 54,2 \\
Sencor & Metribuzin & III & 9 & 18,7 \\
Ridomil & Metalaxil & III & 7 & 14,6 \\
Derosol & Carbendazim & III & 3 & 6,2 \\
Kasumin & Casugamicina & III & 2 & 4,2 \\
Dithane H_45 & Mancozeb & III & 1 & 2,1 \\
Dithane m-22 & Maneb & III & 1 & 2,1 \\
Roundup & Glifosato & IV & 7 & 14,6 \\
\hline
\end{tabular}

Fonte: Visalegis- Anvisa

Tabela 2 - Frequência das variáveis sexo, idade e tempo de trabalho dos $\mathbf{5 0}$ trabalhadores expostos ao organofosforado. Teresópolis/RJ, 2006

\begin{tabular}{lll}
\hline Variáveis & $\mathbf{N}$ & $\%$ \\
\hline Sexo & & \\
Masculino & 20 & 40 \\
Feminino & 30 & 60 \\
Idade & & \\
21-35 anos & 22 & 44 \\
36- 45 anos & 15 & 30 \\
46-59 anos & 13 & 26 \\
Anos de trabalho & & \\
-10 anos & 13 & 26 \\
11-20 anos & 11 & 22 \\
21-50 anos & 26 & 52 \\
\hline
\end{tabular}

Com relação aos anos de exposição, a maioria dos trabalhadores apresentou um longo tempo de exposição a agrotóxicos (21 a 50 anos).

As perguntas realizadas no questionário, que estão contidas na Tabela 3, consistiam nos dados básicos de anamnese clínica realizadas em consulta médica. Observou-se que 38 trabalhadores $(76 \%)$ referiram ter apresentado pelo menos um episódio de tonteira em sua vida e destes, 29 (58\%) trabalhadores ainda sentem tonteira. Atualmente, 27 (54\%) sentem zumbido e 23 (46\%) sentem plenitude auricular.

Dos 50 trabalhadores, 37 (74\%) acreditam possuírem boa audição e mesmo assim, destes, 35 (70\%) sentem dificuldades na compreensão da fala. Estes trabalhadores dizem que escutam, mas não compreendem bem o que falam.

Tabela 3 - Frequência das variáveis sobre a saúde auditiva dos 50 trabalhadores expostos ao organofosforado. Teresópolis/RJ, 2006

\begin{tabular}{lcccc}
\hline Variáveis & Sim & $\%$ & Não & $\%$ \\
\hline Já sentiu tontura & 38 & 76 & 12 & 24 \\
Atualmente, sente tontura & 29 & 58 & 21 & 42 \\
Atualmente, sente zumbido & 27 & 54 & 23 & 46 \\
Atualmente, sente plenitude auricular & 23 & 46 & 27 & 54 \\
Já apresentou alguma doença no ouvido & 16 & 32 & 34 & 68 \\
Acredita que escuta bem & 37 & 74 & 13 & 26 \\
Apesar de escutar bem, sente dificuldade de compreender o que falam & 35 & 70 & 15 & 30 \\
\hline
\end{tabular}




\section{DISCUSSÃO}

Com relação aos agrotóxicos, pode-se observar o uso de vários produtos simultaneamente. Outros estudos revelam que os agrotóxicos mais utilizados pelos agricultores eram aqueles com maior nível de toxicidade ${ }^{8,21}$, corroborando o presente estudo.

Pode-se observar na Tabela 2 que se trata de uma população jovem e em plena atividade profissional, com grande parte da população iniciando no trabalho agrícola desde a infância, seguindo os padrões paternos. Esta prática é mais frequente no período de plantio e colheita sazonal. Os agricultores de pequeno e médio porte parecem conhecer, em menor grau, os perigos para saúde, decorrentes do uso dos agrotóxicos. Eles relatam que, em poucos anos de aplicação dos produtos, precisam contratar os mais jovens para a atividade, pois não se sentem mais em condições de fazê-lo. Segundo os relatos, esses jovens começam a trabalhar em média aos 17 anos e ao completarem 25 anos, ou 26, já não têm mais condições de continuar no serviço ${ }^{22}$. Outro aspecto a ser levado em consideração é que os jovens são afetados pelo trabalho precoce, exposição ao agrotóxico; jornada de trabalho e principalmente o comprometimento do processo de socialização infantil e da escolarização ${ }^{23}$.

Em geral, o tempo das atividades na agricultura tem um início bem precoce na maioria das comunidades rurais brasileiras. Observa-se o ingresso ainda criança, entre 7-8 anos de idade, e o tempo médio estimado de exposição está em torno de 20 anos $^{8}$.

De acordo com a Tabela 3, observou-se que $76 \%$ dos trabalhadores apresentaram, pelo menos, um episódio de tontura, corroborando outro estudo ${ }^{8}$, e muitos, ainda sofrem com o sintoma, podendo significar que a intoxicação é lenta ou que sintomatologia estará presente em função da exposição. Com relação à audição, muitos que relatam escutar bem, sentem dificuldades de compreender as palavras, sugerindo que este sintoma pode ser um sinal neurológico ${ }^{24}$, o que mostra que este dado deve ser investigado com avaliação audiométrica e outros exames objetivos complementares.

Segundo um estudo sobre o uso e manejo dos agrotóxicos ${ }^{25}$, diferentes sintomas são associados a diferentes tipos de agrotóxicos, por outro lado, na análise do estudo transparece que os trabalhadores minimizam a relação do agrotóxico com os sintomas encarando dor de cabeça e problemas de estômago, por exemplo, como processos naturais decorrentes do seu manejo.

As políticas de saúde partem do pressuposto de que existe uma relação direta entre o conhecimento e a prática, tendo suas ações voltadas apenas para a transmissão de informações, cabendo ao indivíduo a responsabilidade do cuidado da saúde. Podese observar que apesar do agricultor ter a informação dos riscos à saúde e do meio ambiente quanto ao uso do agrotóxico, ele continua usando de forma indiscriminada e sem a devida proteção individual. Enquanto os indivíduos não tiverem suas necessidades mais básicas (fisiológicas) atendidas, eles não se preocupariam em atender outras necessidades hierarquicamente superiores ${ }^{26}$. Isso pode explicar porque o produtor rural, na sua decisão de usar ou não agrotóxico, muitas vezes, por questão de sobrevivência, relevaria a um segundo plano, neste curto prazo, os seus efeitos negativos sobre a sua saúde e sobre o meio ambiente. Claro que somente a transmissão do conhecimento técnico para uma população de baixa escolaridade, o rótulo das embalagens com uma linguagem que não está de acordo com a do usuário ${ }^{27}$ deixam a desejar. A ineficácia do treinamento e a falta de vigilância (uso de receituário, venda de produtos ilegais, etc) agravam mais ainda a saúde do agricultor.

São os determinantes sociais (moradia, saneamento, educação, etc) que favorecerão a lógica da saúde e da qualidade de vida, conscientizando e dando ferramentas necessárias ao agricultor na busca de soluções mais adequadas.

Com este estudo, destaca-se que o sistema auditivo e vestibular deva ser avaliado e que alterações possam ser detectadas durante a anamnese e avaliação clínica específica do trabalhador rural, e devidamente pesquisada pelas equipes de saúde responsáveis pelo atendimento médico nas localidades. Ressalta-se, portanto, que são necessárias ações que promovam não só o conhecimento, mas a capacitação desta população para o uso adequado de agrotóxicos, bem como a divulgação de outras formas de cultivo que não necessitem do agrotóxico.

\section{CONCLUSÃO}

A partir da análise dos resultados de 50 entrevistas realizadas em trabalhadores rurais expostos aos agrotóxicos de tipo organofosforados, pode-se observar uma alta prevalência de queixas auditivas e vestibulares, sugerindo que estas substâncias podem oferecer riscos a estes sistemas em função de suas ações ototóxicas e neurotóxicas. É importante a continuidade da condução de outros estudos que avaliem a função auditiva periférica e central em trabalhadores expostos aos agrotóxicos para complemento dos resultados obtidos neste estudo de queixas auditivas e vestibulares. 


\begin{abstract}
Purpose: to characterize the auditory and vestibular symptoms of rural workers under an environmental exposed of organophosphate pesticides. Methods: this is a descriptive epidemic study that evaluated 50 workers. The age group varied from 21 to 59 years with a mean age of 38.3 years. There were 20 $(40 \%)$ male and $30(60 \%)$ female workers. A questionnaire was used with questions related to the auditory health in addition to some specific questions on time exposure. Results: the results showed that $38(76 \%)$ workers showed dizziness and $29(58 \%)$ of them continued showing these symptom; 27 $(54 \%)$ related tinnitus; $23(46 \%)$ fullness sensation. 37 (74\%) workers don't have problem with hearing but $35(70 \%)$ can't understand very well the spoken words. Data suggest that the pesticides induce auditory and vestibular system alterations. It is possible to affirm that it is a slow, insidious and silent intoxication. Conclusions: dizziness and hearing loss are a constant and subclinical symptoms in occupational exposure suggesting a precocious intoxication signal, harming the quality of life of these workers.
\end{abstract}

KEYWORDS: Environmental Exposure; Occupational risks; Inseticides, Organophosphate; Dizziness

\section{REFERÊNCIAS}

1. Comissão das Comunidades Européias. Livro Branco: estratégias para a futura política em matéria de substâncias químicas. Bruxelas: manuscrito traduzido do consenso da comissão das Comunidades Europeias; 2001.

2. Peres F. Os desafios da educação ambiental e em saúde para a saúde pública no limiar do milênio. In: Mata SF, Gavazza S, Almeida MCM, Ottoni AB, organizadores. Educação ambiental, desafio do século: um apelo ético. Rio de Janeiro: Terceiro Milênio. 1998.p.156-62.

3. Jaga K, Dharmani C. Sources of exposure to and public health implications of organophosphate pesticides. Rev Panam Salud Publica. 2003; 14(3):171-85.

4. Leveridge YR. Pesticide poisoning in Costa Rica during 1996. Vet Hum Toxicol. 1998; 40(1):42-4.

5. Gomes J, Lloyd O, Revitt MD, Basha M. Morbidity among farm workers in a desert country in relation to long-term exposure to pesticides. Scand J Work Environ Health. 1998; 24(3):213-9.

6. Ellenhorn MJ. Ellenhorn's medical toxicology: diagnostic and treatment of human poisoning. Medical Toxicology. 2. ed. Baltimore: William \& Wilkins; 1997.

7. Lal CS, Kumar V, Ranjan A, Dias VNR, Kumar N, Kishore K, et al. Evaluation of cholinesterase level in an endemic population exposed to malathion suspension formulation as a vector control measure. Mem Inst Oswaldo Cruz. 2004; 99(2):219-21.

8. Araújo AJ, Lima JS, Moreira JC, Jacob SC, Soares MO, Monteiro MCM, et al. Exposição múltipla a agrotóxicos e efeitos à saúde: estudo transversal em amostra de 102 trabalhadores rurais, Nova Friburgo, RJ. Ciênc Saúde Colet. 2007; 12(1):115-30.

9. Delgado IF, Paumgarten FJR. Intoxicações e uso de pesticidas por agricultores do Município de Paty do Alferes, Rio de Janeiro, Brasil. Cad Saúde Pública. 2004; 20(1):180-6.

10. Carod-Artal FJC, Speck-Martins A. Polineuropatía tardía inducida por exposición a organofosforados. Rev Neurol. 1999; 29(2):123-7.

11. Sivayoganathan S, Gnanachandran J, Lewis Fernando $M$. Protective measure use and symptoms among agropesticide applicatons in Sri Lanka: health and social sciences in Sri Lanka. An overview of the triangle programme. Soc Sci Med. 1995; 40(4):431-6.

12. Richter ED, Chuwers P, Levy Y, Gordon M, Grauer F, Marzouk J, et al. Health effects from exposure to organophosphate pesticides in workers and residents in Israel. Isr J Med Sci. 1992; 28(8-9):584-98.

13. Chen SY, Zhang ZW, He FS, Yao PP, Wu $Y Q$, Sun JX, et al. An epidemiological study on occupational acute pyrethroid poisoning in cotton farmers. Br J Ind Med. 1991; 48(2):77-81.

14. He F, Sun J, Han K, Wu Y, Yao P, Wang S, et al. Effects of pyrethroid insecticides on subjects engaged in packaging pyrethroids. $\mathrm{Br} \mathrm{J}$ Ind Medicine. 1988; 45(8):548-51.

15. Gupta RC, Dave SK, Shah MP, Kashyap SK. A monitoring study of workers handling pesticides in warehouses and godowns. J Environ Sci Health B. 1979; 14(4):405-16.

16. Quinones MA, Bogden JD, Louria DB, Nakah AE, Hansen C. Depressed cholinesterase activities among farm workers in New Jersey. Sci Total Environ. 1976; 6(2):155-9. 
17. Hülse M, Federspil P. [Disturbance of equilibrium due to poisoning with organophosphate pesticides (author's transl)]. HNO. 1975; 23(6):185-9.

18. Pacheco-Ferreira H, Filhote MIF, Haikel S, Noronha C, Carvalho TA. Monitoramento dos riscos e efeitos a saúde de agentes comunitários expostos ocupacionalmente aos organofosforados: estudo ocupacional, clínico e neuropsicológico. Cad Saúde Colet. 2000; 8(1):27-38.

19. Manjabosco CW, Morata TC, Marques JM. Perfil audiométrico de trabalhadores agrícolas. Arq Otorrinolaringol. 2004; 8(4):284-95.

20. Martin CO, Adams Junior HP. Neurological aspects of biological and chemical terrorism: a review for neurologists. Arch Neurol. 2003; 60(1):21-5.

21. Soares W, Almeida RMVR, Moro S. Trabalho rural e fatores de risco associados ao regime de uso de agrotóxicos em Minas Gerais, Brasil. Cad Saúde Pública. 2003; 19(4):1117-27.

22. Gomide M. Agrotóxico: que nome dar? Ciênc Saúde Colet. 2005; 10(4):1047-54.
23. Silva JM, Novato-Silva E, Faria HP, Pinheiro TMM. Agrotóxico e trabalho: uma combinação perigosa para a saúde do trabalhador rural. Ciênc Saúde Colet. 2005; 10(4):891-903.

24. Teixeira CF, Augusto LGS, Morata TC. Occupational exposure to insecticides and their effects on the auditory system. Noise Health. 2002; 4(14):31-9

25. Fonseca MGU, Peres F, Firmo JOA, Uchôa E. Percepção de risco: maneiras de pensar e agir no manejo de agrotóxicos. Ciênc Saúde Colet. 2007; 12(1):39-50

26. Maslow $\mathrm{AH}$. Theory of human motivation. Psychol Rev. 1943; (50):370-96.

27. Bedor CNG, Ramos LO, Pereira PJ, Rêgo MAV, Pavão AC, Augusto LGS. Vulnerabilidades e situações de riscos relacionados ao uso de agrotóxicos na fruticultura irrigada. Rev Bras Epidemiol. 2009; 12(1):39-49.

RECEBIDO EM: 16/09/2008

ACEITO EM: 24/06/2009

Endereço para correspondência:

Ana Cristina Hoshino

Rua Geminiano Góis, 170/802

Rio de Janeiro - RJ

CEP: 22743-670

E-mail: choshino@uol.com.br 